\title{
Optical Theorem and Effective Finite-Range Nuclear Interaction for Low-Energy Nuclear-Fusion Reactions
}

\author{
Y. E. Kim and A. L. ZuBAREv \\ Department of Physics, Purdue University - West Lafayette, IN 47907 - 1396, USA
}

(Nuovo Cimento A, 108 (1995) 1009)

PACS 24.10 - Nuclear reaction and scattering models and methods.

PACS 25.70.Jj - Fusion and fusion-fission reactions.

PACS 99.10 - Errata.

$Q$ values used in this paper are from old mass tables (Nucl. Phys., 18 (1960) 529 and Nuclear Wallet Cards, July 1990, published by J. K. Tuli, National Nuclear Data Center for the U.S. Nuclear Data Network) and hence some values are obsolete. In the following, we present new modified sentences which include the updated $Q$ values calculated from mass differences given in the latest Nuclear Wallet Cards (July 1995, fifth edition).

The sentences appearing in last four rows of page 1021 and the first eight rows of page 1022 contain incorrect $Q$ values and should be replaced with the following sentences:

For ${ }^{A} \mathrm{Pd}(\mathrm{d}, \mathrm{p})^{A+1} \mathrm{Pd}, Q$ values are $5.4 \mathrm{MeV}(A=102), 4.87 \mathrm{MeV}(A=104)$, $7.34 \mathrm{MeV}(A=105), 4.31 \mathrm{MeV}(A=106), 7.0 \mathrm{MeV}(A=107), 3.9 \mathrm{MeV}(A=108)$, and $3.53 \mathrm{MeV}(A=110)$. For tritium producing ${ }^{A} \mathrm{Pd}(\mathrm{d}, \mathrm{t})^{A-1} \mathrm{Pd}$ reactions, all $Q$ values are negative for stable ${ }^{A} \mathrm{Pd}(A=102,104,105,106,108$ and 110). However, there are other tritium producing reactions with positive $Q$ values such as ${ }^{6} \mathrm{Li}(\mathrm{d}, \mathrm{t})^{5} \mathrm{Li}(Q=0.592)$ $\left({ }^{5} \mathrm{Li}\right.$ decays to ${ }^{4} \mathrm{He}+\mathrm{p}$ with $Q=1.97 \mathrm{MeV}$ and $\left.\Gamma=1.5 \mathrm{MeV}\right)$. If X-rays or bremsstrahlung radiation is not observed or is at a very low level in the electrolysis experiments [3], all of the above fusion reactions involving $\mathrm{Pd}$ isotopes are ruled and hence we need to look for other candidate fusion reactions with small values of $Q$, including those involving impurity isotopes in electrolysis experiments. Examples of other possible fusion reactions with small $Q$ values are ${ }^{102} \mathrm{Pd}\left({ }^{7} \mathrm{Li},{ }^{6} \mathrm{Li}\right){ }^{103} \mathrm{Pd}$ $(Q=0.375 \mathrm{MeV}),{ }^{108} \mathrm{Pd}\left({ }^{14} \mathrm{~N},{ }^{15} \mathrm{~N}\right){ }^{107} \mathrm{Pd}(Q=7.6 \mathrm{MeV}),{ }^{110} \mathrm{Pd}\left({ }^{6} \mathrm{Li},{ }^{5} \mathrm{Li}\right){ }^{111} \mathrm{Pd} \quad(Q=0.086$ $\mathrm{MeV})$, etc. 